\title{
Multicampi Saúde: vivências de acadêmicos de enfermagem no Munícipio de \\ Castanhal - Pará
}

Multicampi Saúde: experiences of nursing students in the Municipality of Castanhal - Pará

Multicampi Saúde: experiencias de estudiantes de enfermería em el Municipio de Castanhal - Pará

Marcelle Lorena Figueira Noronha ORCID: https://orcid.org/0000-0002-6182-0083 Universidade Federal do Pará, Brasil

E-mail: marcellenoronhac1@gmail.com

Leila Cristina da Silva Martins ORCID: https://orcid.org/0000-0002-9068-4914 Universidade Federal do Pará, Brasil E-mail: leilalenf@gmail.com

Diego Pereira Rodrigues ORCID: https://orcid.org/0000-0001-8383-7663 Universidade Federal do Pará, Brasil

E-mail: diego.pereira.rodrigues@gmail.com

Marília de Fátima Vieira de Oliveira ORCID: https://orcid.org/0000-0003-4303-9434 Universidade Federal do Pará, Brasil E-mail: mariliafvo@ufpa.br

Roseneide dos Santos Tavares ORCID: https://orcid.org/0000-0003-4556-2683 Universidade Federal do Pará, Brasil E-mail: rstavares@ufpa.br

Débora Talitha Neri

ORCID: https://orcid.org/0000-0001-6658-2304 Universidade Federal do Pará, Brasil

E-mail: tathaneri@gmail.com

Angelson Camargo Gomes

ORCID: https://orcid.org/0000- 0003-3914-8603 Universidad Privada del Este, Paraguai E-mail: angelson_17@hotmail.com

João Lucas Moraes Souza

ORCID: https://orcid.org/0000-0003-3652-751X Universidade Federal do Pará, Brasil

E-mail: jlucasmsouza@gmail.com

Luenne Chaves de Oliveira ORCID: https://orcid.org/0000-0002-1429-4519 Universidade Federal do Pará, Brasil E-mail: luenneolive@gmail.com

Ana Luísa Soares dos Santos

ORCID: https://orcid.org/0000-0002-3216-1111 Universidade Federal do Pará, Brasil E-mail: analuisa.soares98@gmail.com

Elisângela da Silva Ferreira

ORCID: https://orcid.org/0000-0002-2506-1622 Universidade Federal do Pará, Brasil E-mail: licalipe8@yahoo.com.br

\begin{abstract}
Resumo
O Multicampi Saúde é um projeto de extensão da Universidade Federal do Pará (UFPA) que busca fortalecer a formação profissional dos alunos de graduação dos cursos da área da saúde e afins para integrá-los aos serviços de saúde, em particular, na área da Atenção Básica, com foco na política de atenção à saúde da criança. Objetivo: relatar as experiências vivenciadas por acadêmicas de enfermagem durante atividades de extensão realizadas em um município do Estado do Pará. Metodologia: estudo descritivo, do tipo relato de experiência, desenvolvido por duas alunas do curso de enfermagem integrantes de um Multicampi Saúde, desenvolvidas em Castanhal, no estado do Pará, no mês de novembro de 2019. Resultados: as atividades foram desenvolvidas a partir de um plano de trabalho elaborado pelas preceptoras a partir do acompanhamento de uma "criança-guia" e sua família, além dos demais usuários do Sistema Único de Saúde (SUS). As atividades realizadas foram: consulta de enfermagem, visita domiciliar e ações educativas para a comunidade e equipe de saúde. A participação em uma equipe multiprofissional possibilitou
\end{abstract}


a interação entre os profissionais e assistência e cuidado mais abrangente dos usuários. Considerações Finais: a integração entre ensino e serviço proporciona melhor capacitação do docente, do estudante e do profissional do serviço de saúde, sendo uma importante estratégia para alcançar as mudanças no processo formativo dos acadêmicos e futuro profissionais de saúde.

Palavras chaves: Enfermagem; Acadêmicos; Atenção básica; Multiprofissional; Ensino-serviço.

\begin{abstract}
Multicampi Saúde is an extension project of the Federal University of Pará (UFPA) that seeks to strengthen the professional training of undergraduate students in health courses and the like to integrate them into health services, particularly in the area of Attention Basic, focusing on child health care policy. Objective: to report how experiences lived by nursing students during extension activities carried out in a municipality in the State of Pará. Methodology: a descriptive study, of the experience report type, developed by two nursing students from a Multicampi Saúde, developed in Castanhal, in the state of Pará, in the month of November 2019. Results: the activities were developed based on a work plan prepared by the tutors based on the monitoring of a "guide child" and his family, in addition to the other users of the Unified Health System (SUS). The activities carried out were: nursing consultation, home visit and educational actions for the community and the health team. Participation in a multiprofessional team enabled interaction between professionals and broader assistance and care from users. Final Considerations: an integration between teaching and service offers the best training for teachers, students and health service professionals, being an important strategy to achieve changes in the training process of academics and future health professionals.
\end{abstract}

Keywords: Nursing; Academics; Basic attention; Multi-professional; Teaching-service.

\title{
Resumen
}

Multicampi Saúde es un proyecto de extensión de la Universidad Federal de Pará (UFPA) que busca fortalecer la formación profesional de estudiantes de pregrado en cursos de salud y afines para integrarlos a los servicios de salud, particularmente en el área de Atención. Básico, centrado en la política de salud infantil. Objetivo: reportar cómo vivieron los estudiantes de enfermería durante las actividades de extensión realizadas en un municipio del Estado de Pará. Metodología: estudio descriptivo, del tipo relato de experiencia, desarrollado por dos estudiantes de enfermería de un Multicampi Saúde, desarrollado en Castanhal, en el estado de Pará, en el mes de noviembre de 2019. Resultados: las actividades se desarrollaron en base a un plan de trabajo elaborado por los tutores a partir del seguimiento de un "niño guía" y su familia, además de los demás usuarios del Sistema Único de Salud (SUS). Las actividades realizadas fueron: consulta de enfermería, visita domiciliaria y acciones educativas para la comunidad y el equipo de salud. La participación en un equipo multiprofesional permitió la interacción entre los profesionales y una mayor asistencia y atención por parte de los usuarios. Consideraciones finales: una integración entre docencia y servicio ofrece la mejor formación para docentes, estudiantes y profesionales de los servicios de salud, siendo una estrategia importante para lograr cambios en el proceso de formación de académicos y futuros profesionales de la salud.

Palabras clave: Enfermería; Académica; Atención básica; Multi-profesional; Docencia-servicio.

\section{Introdução}

O Multicampi Saúde é um projeto de extensão da Universidade Federal do Pará (UFPA), como atividade transversal ao ensino, que busca fortalecer a formação profissional dos alunos de graduação participantes, tendo em vista que as ações programadas possibilitam integrá-los, ainda no período de formação profissional, aos serviços de saúde, em particular, na área da Atenção Básica, com foco na política de atenção à saúde da criança (Miyake, 2019).

O Ministério da Saúde, na Portaria no 2.436 de 21 de setembro de 2017 (Brasil, 2017), que aprova a Política Nacional de Atenção Básica (PNAB), considera similares os termos “Atenção Básica (AB)” e “Atenção Primária à Saúde (APS)". Portanto, neste artigo optou-se por utilizar o termo Atenção Básica em todo o texto.

A Atenção Básica é o conjunto de ações de saúde individuais, familiares e coletivas que envolvem promoção, prevenção, proteção, diagnóstico, tratamento, reabilitação, redução de danos, cuidados paliativos e vigilância em saúde, desenvolvida por meio de práticas de cuidado integrado e gestão qualificada, realizada com equipe multiprofissional e dirigida à população em território definido, sobre as quais as equipes assumem responsabilidade sanitária (Brasil, 2017).

Com isso, o Multicampi visa o trabalho em equipe multiprofissional na Atenção Básica. Entende-se que os desafios presentes nos serviços da Atenção Básica, como o desenvolvimento da colaboração e a integração entre diversos núcleos de 
saberes e práticas, podem ser solucionados ou amenizados pela aproximação dos profissionais nas equipes através do estreitamento de vínculo entre eles (Rocha et al., 2020).

A atuação multiprofissional no Multicampi é uma das formas de colaboração para a reflexão sobre a prática do público-alvo, utilizando-se de entrevistas individuais e coletivas com os profissionais de saúde, rodas de conversa com usuários, confecção de diário de campo, capacitações e a utilização de bancos de dados secundários do Sistema Único de Saúde - SUS (Miyake, 2019).

Visando a interação e atuação multiprofissional, participaram deste Projeto alunos de dez (10) cursos de graduação da UFPA: medicina, enfermagem, fisioterapia, terapia ocupacional, odontologia, farmácia, nutrição, biomedicina, psicologia e serviço social. As ações do Projeto foram programadas e implantadas em Belém e outros quatro municípios do estado do Pará: Cametá, Castanhal, Abaetetuba e Bragança.

Sendo assim, a interdisciplinaridade do projeto vem com o intuito de gerar trocas entre os especialistas, através de diálogo e trabalho em equipe, a fim de alcançar o desenvolvimento de um pensamento que responda pela complexidade da saúde do indivíduo (Miyake, 2019).

Conforme Justus Neto et al. (2017), a interdisciplinaridade se caracteriza pela interação de diversas disciplinas que atuam dentro de um mesmo projeto. O objetivo é o enfrentamento e resolução dos problemas, não apenas uma simples troca de informação. Para esses autores, a interdisciplinaridade deve desencadear um reconhecimento dos limites e das potencialidades de cada campo de saber. Na saúde, o termo interdisciplinar precisa ser interpretado como uma maneira de abordar determinadas situações ou problemas, integrando e articulando diferentes conhecimentos e práticas, valorizando o entendimento e as atribuições de cada categoria profissional.

É evidente a importância do protagonismo do enfermeiro na Atenção Básica. Conforme a Lei do Exercício Profissional, $\mathrm{n}^{\circ}$ 7.498, o enfermeiro assume diferentes modelos assistenciais e atividades indispensáveis, dentre elas: planejamento, execução e avaliação dos serviços, prescrição medicamentosa, consultas e solicitação de exames (Brasil, 1986).

Sobre a atuação do enfermeiro na Atenção Básica no Brasil, Ferreira et al. (2018) afirmam que este vem se constituindo como um instrumento de mudanças nas práticas de atenção à saúde no SUS, respondendo a proposta do novo modelo assistencial que não está centrado na clínica e na cura, mas, sobretudo, na integralidade do cuidado, na intervenção frente aos fatores de risco, na prevenção de doenças e na promoção da saúde e da qualidade de vida.

Com isso, percebe-se que a enfermagem possui uma grande importância na equipe multiprofissional, pois há a necessidade de uma relação interdisciplinar com os outros profissionais para que os objetivos esperados para o cuidado integral ao paciente sejam alcançados.

No que tange à formação do enfermeiro para atuação multiprofissional, Brito et al. (2017) aponta que articular saberes, dentro dos diversos cenários de aprendizagem é contemplar a relação entre o ensino e o serviço, assim como entre a teoria e a prática. A formação do enfermeiro deve estar pautada para a atuação na integralidade da atenção à saúde, na construção de vínculos entre profissionais e usuários e na superação do modelo centralizador persistente (existente) até os dias atuais.

Portanto, compreende-se que se torna valiosa a emancipação do discente e docente ao participar de um projeto voltado para a área da saúde com o intuito de entrelaçar saberes, tais como: saberes práticos, subjetivos, objetivos, teórico-científicos, entre outros. O que sugere a capacitação destes para a qualificação do serviço que será prestado e usar como exemplo os aprendizados para futuros trabalhos e também para futuros profissionais.

Esse estudo ocupa sua significância junto à comunidade científica a partir do momento que divulga seus resultados, suas experiências e as mudanças positivas no cenário da atenção básica. Traz ainda a oportunidade de dar continuidade e/ou gerar novos projetos com essa mesma característica de atenção à saúde. Além da finalidade de incentivar docentes, discentes e 
instituições a executá-lo em outros locais do país e assim poder acrescentar conteúdo prático-científico para o fortalecimento do atuar na profissão durante a prática dos discentes e docentes quanto à prática-pedagógica.

Foram várias as motivações que nos levaram a realizar esse estudo, porém viver o dia a dia do serviço com a oportunidade de compreender melhor a organização do processo de trabalho do enfermeiro com a equipe de atenção primária a saúde, foi a maior motivação. Entendemos que o estudante, ao se envolver de forma integral com novas idéias, práticas, culturas, amplia seu olhar para a aprendizagem. Além disso, é importante ressaltar que o conteúdo construído servirá como produção acadêmica que contribuirá e incentivará a realização de trabalhos como esse.

Para tanto, ao longo do estudo surgiram as seguintes questões norteadoras: qual a colaboração de acadêmicos de enfermagem nos serviços de saúde no município de Castanhal-Pará, durante o programa Multicampi? Quais atividades desempenhadas pelos acadêmicos durante a atuação no Programa? Qual a contribuição da participação no Programa para o desenvolvimento profissional dos acadêmicos? Qual a importância da participação no Programa para o cuidado da comunidade atendida, na visão dos acadêmicos?

Assim, traçamos como objetivo para esse estudo: relatar as experiências vivenciadas por acadêmicas de enfermagem durante atividades de extensão realizadas em um município do Estado do Pará.

\section{Metodologia}

Estudo descritivo, de natureza qualitativa, do tipo relato de experiência, desenvolvido por integrantes de um projeto de extensão intitulado Multicampi Saúde. Os estudos qualitativos são aqueles nos quais é importante a interpretação por parte do pesquisador com suas opiniões sobre o fenômeno em estudo (Pereira et al., 2018).

O relato de experiência é uma narrativa científica e modalidade de cultivo de conhecimento no território da pesquisa qualitativa, concebida na reinscrição e na elaboração ativada através de trabalhos da memória, com apresentação das compreensões a respeito do vivido (Daltro \& Faria, 2019).

As atividades de extensão foram desenvolvidas em Castanhal, no estado do Pará, no mês de novembro de 2019, em (04) quatro Unidades Saúde da Família (USF) do município: USF João Câncio Sampaio, USF José Olimpio de Brito, USF Sarah Martins e USF Dr. Luis Charlet.

Este estudo foi realizado por duas acadêmicas cursando o último ano de graduação do curso de enfermagem da UFPA, que atuaram na condição de bolsistas do Projeto de Extensão, com a participação de docentes do curso que operaram como supervisores no município de Castanhal.

Como fontes informações, foram utilizados os relatórios de atividades, construído diariamente pelas alunas, contendo detalhadamente o registro de todas as atividades propostas no plano de trabalho, incluindo comentários e reflexões acerca das suas atuações no contexto do Projeto.

Além disso, foi utilizado o diário de campo, com registro individual das atividades realizadas, onde constavam as atividades realizadas e pontos a destacar da vivência do dia, além das dificuldades encontradas e os pontos positivos das ações desenvolvidas, bem como outros comentários pertinentes.

Este estudo por não envolver pessoas diretamente nesse processo dispensou-se o Termo de Consentimento Livre e Esclarecido, porém, manteve-se o respeito aos valores éticos. 


\section{Resultados e Discussão}

O projeto Multicampi Saúde, tem como foco a Política de Atenção à Saúde da Criança. Devido isso, inicialmente, é aplicado um curso introdutório na Política Nacional de Atenção Integral à Saúde da Criança (PNAISC) para os docentes supervisores, preceptores e discentes dos cursos selecionados com auxílio da Coordenação Estadual de Saúde da Criança e Coordenação Geral de Saúde da Criança/Ministério da Saúde.

A PNAISC tem por objetivo promover e proteger a saúde da criança e o aleitamento materno, mediante a atenção e cuidados integrais e integrados da gestação aos 9 (nove) anos de vida, com especial atenção à primeira infância e às populações de maior vulnerabilidade, visando à redução da morbimortalidade e um ambiente facilitador à vida com condições dignas de existência e pleno desenvolvimento (Brasil, 2015).

Embora o objetivo principal do Projeto Multicampi seja direcionar a atuação na saúde da criança, faz-se necessário o conhecimento e análise do ambiente familiar em que a criança está inserida e identificar possíveis problemas que possam afetar sua saúde.

Fazem parte do Projeto, além dos discentes participantes, docentes supervisores e preceptores. Os docentes são aqueles responsáveis pelo andamento do projeto e por acompanhar as atividades desenvolvidas no período em que estiver ocupando o cargo. Estes são docentes da Universidade Federal do Pará, provenientes dos cursos da saúde selecionados para participar, havendo uma troca semanal de supervisores. Os preceptores por sua vez, são os profissionais de saúde que atuam no município.

Sendo que participação destes é de suma importância, pois atuam integrados à educação como atores permanentes e atualizados quanto às melhores evidências da área da saúde em que exercem suas atividades. Buscando assim contribuir com o aprendizado significativo de competências e habilidades para planejar, desenvolver e avaliar práticas multidimensionais em saúde.

Antes do início de cada mês de atividades, supervisores e preceptores formam grupos de discentes participantes com o objetivo de alocar em cada Unidade de Saúde um aluno de cada curso de graduação, permanecendo nesta pelo período de um mês, atuando diariamente com os profissionais que prestam serviços nos mesmos. Os discentes recebem uma bolsa-auxílio, além de alojamento e alimentação, fornecidos pelo Projeto, durante todo o período de duração do programa.

As atividades são desenvolvidas a partir de um plano de trabalho elaborado pelas preceptoras para atuação em território adscrito à Unidade de Saúde onde os alunos foram alocados, juntamente com os membros da equipe de saúde. Posteriormente, é feito o reconhecimento das especificidades e funcionamento da mesma, para tanto, utiliza-se a base de dados secundários dos indicadores e da rede de Atenção Básica do município, fornecida pela secretaria de Saúde. Esta atividade permite o reconhecimento de demanda populacional pela qual as unidades são responsáveis, as principais demandas de saúde de cada área, para assim estabelecer uma melhor visão geral, buscando um foco de atividades a serem desenvolvidas.

Durante o projeto cada discente recebe uma "criança-guia", que será atendida por ele até o término do período de atuação. Ela possui essa nomenclatura por ser considerada a guia dos profissionais de saúde até suas famílias, pois através de suas demandas identificadas pelos Agentes Comunitários de Saúde (ACS) e por outros profissionais da Unidade, receberão atendimento integral juntamente com seus familiares.

Para tanto, são desenvolvidas atividades específicas direcionadas à saúde da criança como: visita domiciliar ao recémnascido; consulta de enfermagem e acompanhamento da puérpera e recém-nascido na primeira semana pós-parto; consultas subsequentes do recém-nascido; busca ativa de crianças vulneráveis; atualização da caderneta da criança e calendário vacinal; realização do teste do pezinho; acompanhamento e desenvolvimento de crianças de 0 a 5 anos; ações integradas nas doenças prevalentes na infância - AIDPI em crianças de 0 a 5 anos; orientação e estímulo ao aleitamento materno. 
Ao compreender que a importância da saúde do adulto que convive e cuida da criança, os acadêmicos de enfermagem integrantes do Multicampi Saúde realizam, também, atividades voltadas para a família, como: consulta de enfermagem nos Programas de Saúde, ações de educação em saúde para a comunidade/usuários e profissionais de saúde, participação em reuniões como a equipe de saúde da Unidade e em atividades programadas pela equipe multiprofissional.

A atuação do acadêmico de enfermagem no Projeto está pautada na competência do enfermeiro na Atenção Básica, como: realizar assistência integral às pessoas e famílias na Unidade Básica de Saúde (UBS), nos domicílios e espaços comunitários; realizar consultas de enfermagem, solicitar exames complementares e prescrever medicações, observadas as disposições legais da profissão e conforme os protocolos ou outras normativas técnicas; planejar, gerenciar, coordenar e avaliar as ações desenvolvidas pelos ACS (Sousa et al., 2020).

Sobre isso, Lopes et al. (2020) afirma que o aprimoramento de competências pode ser iniciado durante a formação acadêmica dos profissionais e, em continuidade, cabe ao enfermeiro buscar constantemente conhecimentos para sua atuação. Para isso, deve-se utilizar o pensamento crítico-reflexivo, levando em consideração as necessidades de saúde da população.

Durante o período de atuação, observou-se o engajamento dos profissionais de saúde das Unidades em ofertar a melhor forma de assistência possível, mesmo com limitações do sistema. Além disso, a vivência diária nas Unidades nos proporcionou momentos especiais e de grande aprendizado.

Verificou-se que a ida da criança à unidade de saúde está pautada na realização de consultas de rotina e vacinação. Quanto às consultas de enfermagem no Programa de puericultura, realizadas em um dia na semana, observou-se que a maioria das crianças atendidas não apresentava grandes problemas de saúde, porém, a importância em manter as consultas em dia com foco na promoção e prevenção da saúde foi perceptível e compatível com o preconizado pelas políticas de saúde. Conforme Brito et al. (2018), o programa de puericultura na Atenção Básica institui-se em um conjunto de medidas e cuidados preventivos capazes de orientar a promoção da saúde e o bem-estar da criança, refletindo-se sobre o desenvolvimento nos aspectos físico, emocional e social.

Durante as consultas de puericultura foi possível realizar exame físico em todas as crianças, assim como o preenchimento da caderneta de saúde e realização de procedimentos atrasados, tais como administração de vitamina $\mathrm{A}$, aprazamento de vacinação, e até encaminhamento para outros profissionais e/ou localidades com serviços específicos. Sendo possível a identificação de possíveis diagnósticos médicos como escabiose e coqueluche, sendo devidamente encaminhados aos especialistas, com adequada resolutividade.

Foi notória a importância da consulta de enfermagem neste Programa, sendo de grande enriquecimento para os conhecimentos científicos e técnicos das mesmas. Entende-se que a puericultura desempenha importante papel no fortalecimento da assistência, para que se reduzam índices de morbidade e mortalidade, graças à detecção precoce de problemas de saúde e prescrição de cuidados, além da implementação de ações interventivas para melhoria da qualidade do atendimento prestado aos usuários (Brito et al., 2018).

Em relação às visitas domiciliares, estas foram realizadas às mais diversas configurações de famílias. Houve a oportunidade de conhecer diferentes contextos socioeconômicos ao visitar os lares da população adstrita à Unidade. Logo, entendemos que esta ferramenta é uma das mais importantes na consolidação dos serviços prestados à comunidade.

Uma vez que estar dentro do lar das famílias possibilita observarmos a realidade das mesmas e desta forma obter dados da real necessidade dos cuidados de saúde proporcionando uma intervenção mais eficaz de acordo com a singularidade de cada família. Para a realização deste processo, foi necessária a manutenção do conhecimento sobre comunicação terapêutica do enfermeiro com a família, visto que cada visita se dava de forma diferenciada e em cada família havia diversas necessidades, todas necessitando de formas diferentes de atendimentos às suas necessidades do momento. 
Nesse contexto, ressalta-se a intervenção realizada com as crianças-guias. Inicialmente foi realizada a leitura dos prontuários dessas crianças e levantadas as demandas pontuadas pelos profissionais que as haviam acompanhado anteriormente. Entretanto, foi durante as visitas domiciliares que houve uma observação real das suas necessidades, não apenas relacionada à saúde física, mas também de ordem psicológica, nutricional, etc.

Dentre os problemas identificados podem ser pontuados: luto pela perda da genitora, questões alimentares, déficit no auto-cuidado, rotina familiar desregulada, entre outros. A partir disso foram traçados planos de cuidados e intervenções.

Os momentos vivenciados nos fazem pensar sobre a importância da vista domiciliar, sendo possível observar a complexidade das problemáticas existentes naquele local. Com isso, percebe-se que o acompanhamento da criança não consegue se esgotar na consulta realizada na Unidade, pois envolve outros tipos de questionamentos e necessidade de conhecimentos, como escuta qualificada, observação das condições de saúde e vínculo social com a família.

Conforme Menezes et al. (2019), corroborando com as atividades realizadas no Multicampi, as ações do enfermeiro devem ser voltadas para um cuidado que envolva as demandas e especificidades da criança a partir de seu contexto familiar e social, visando uma assistência à saúde que permita um olhar holístico a partir de um acompanhamento periódico e organizado de maneira sistemática.

Um dos pontos essenciais para o bom andamento do Projeto diz respeito à atuação do grupo multiprofissional, pois a análise e atuação interdisciplinar contribuíram para a interação entre os acadêmicos de diversos cursos, além de um acompanhamento da criança-guia de forma mais abrangente e maior possibilidade de resolução de problemas.

O trabalho multidisciplinar necessita de articulação e dinâmica entre os profissionais, visando um cuidado integral ao paciente. A organização de trabalho, proposta pela Atenção Básica, aponta para a necessidade de um trabalho em equipe, uma vez que a junção dos olhares de diferentes categorias profissionais favorece a interdisciplinaridade, o que interfere positivamente na resolubilidade dos problemas de saúde existentes na comunidade assistida, além de proporcionar uma atenção integral aos indivíduos (Rocha et al., 2020; Barreto et al., 2019).

Contudo, observou-se que a inserção de acadêmicos de diversos cursos da área da saúde e afins propostas pelo Multicampi atende as exigências de um trabalho pautado na interdisciplinaridade e com vistas ao acompanhamento integral dos usuários e família.

Durante as atividades realizadas no Projeto algumas visitas domiciliares à puérperas e recém-nascidos $(\mathrm{RN})$ também foram realizadas. Observou-se a importância de conhecimento sobre aleitamento materno e cuidados com o recém-nascido, visto que havia muitos questionamentos e dúvidas com necessidade de orientações, como pega correta e posicionamento na amamentação, ordenha e cuidados com o RN. Além disso, a visita é importante para que possamos observar o comportamento e vínculo inicial entre mãe e filho, estimular o desenvolvimento da criança com afeto, orientar quanto ao aleitamento materno exclusivo até os seis meses e avaliar a condição da puérpera, tanto física quanto emocional, proporcionando uma intervenção de acordo com as suas necessidades.

A visita no domicílio também proporcionou espaço para a realização de exame físico da puérpera e do RN, bem como avaliação dos reflexos primitivos e mensuração antropométrica do bebê.

Além das consultas de enfermagem e visitas domiciliares, diversas atividades foram realizadas, sendo a grande maioria delas pautadas na educação em saúde, seja dos usuários ou equipe de saúde, elaboradas e direcionadas pelo grupo de discentes multiprofissional, focadas em identificação de problemas e necessidades. Sobre isso, Barreto et al. (2019) afirma que quando essas práticas de educação em saúde são desenvolvidas pela equipe multiprofissional, acabam por incluir uma maior diversidade de saberes, contribuindo para a criatividade e a maior adesão dos usuários. Para eles, esse fato, juntamente ao saber descentralizado do profissional, constitui-se em estratégias estruturais, para tornar as atividades educativas em espaços de partilha de saberes. 
Uma das propostas do Multicampi Saúde é a realização de ações educativas para usuários e equipe de saúde das unidades de saúde dos municípios sede do Projeto. As práticas de educação em saúde, além de estimular mudanças nos comportamentos de riscos dos indivíduos, servem como norte para a reflexão da população, pois proporcionam uma assistência integral e um caráter transformador, por tornarem os usuários ativos no que diz respeito à saúde e autonomia, permitindo-os repensar sobre a realidade em que vivem e optarem por escolhas mais saudáveis (Barretos et al., 2019).

Para os usuários, foram oferecidas diversas atividades em sala de espera dos consultórios e com os grupos de acompanhamento na Unidade com o intuito de gerar debates e reflexões com a comunidade. Nessa perspectiva, foram abordados temas como: alimentação e nutrição, prevenção de acidentes, síndromes hipertensivas na gravidez e direitos do usuário do SUS.

Abordando temas para grupos específicos, foram realizados momentos de acolhimento com o grupo de idosos, abordando a práticas de carinho com o próximo e auto-cuidado, e ação com usuários cadastrados no programa de saúde mental, ressaltando a importância do grupo de apoio para a manutenção de uma boa saúde mental. Para um grupo de gestantes, foi realizada atividade apresentando a importância do pré-natal para sua saúde e do seu bebê. O grupo em tratamento do tabagismo participou de dinâmicas para abordar a diminuição de danos com o controle do tabaco e processo de desmame, objetivando o estímulo de manutenção e assiduidade no grupo.

As ações educativas também se estenderam para além da Unidade de Saúde, a fim de buscar atender os moradores da área coberta pela Unidade e que pouco a frequentam. Para tanto, houve a participação no Programa Saúde na Escola (PSE), com atividades abordando a temática do bullying e gravidez na adolescência, especialmente para adolescentes matriculados em uma escola próxima à Unidade.

É fundamental estabelecer um modelo de capacitação que promova a atenção integral à saúde e, assim, respeite a subjetividade do usuário. Essas estratégias possibilitam a transformação do processo de trabalho, podem produzir mudanças e reflexões, bem como aprimorar a sua prática e se tenham profissionais mais capacitados (Lopes et al., 2020)

Quanto às ações educativas para os profissionais, observou-se a importância em discussões contínuas com a equipe. Com isso, entende-se que se faz necessária a capacitação de profissionais, de forma periódica na forma de educação continuada, especialmente para os agentes comunitários de saúde. Essa observação se dá pelo fato de que a maioria dos ACS não possui formação específica na área da saúde e, portanto, sem conhecimento e habilidades suficientes para o acompanhamento dos usuários e atividades que realizam, além de que, há recentes contratações de novos profissionais, com renovação contínua do quadro de funcionários.

A Educação Permanente em Saúde objetiva a transformação no cenário de prática e trabalho. Deve-se partir da reflexão crítica dos profissionais diante do cotidiano dos serviços para a busca de soluções em conjunto com a equipe para os problemas encontrados. Isso se dá por meio de ações educativas atreladas a capacitações pontuais, de estilo programático e centralizador, com conteúdo padronizado e atualização de conhecimentos de acordo com as especificidades de cada categoria (Ferreira et al., 2019).

Dentre as ações educativas realizadas com os profissionais, foram discutidos temas sugeridos pela própria equipe de saúde, de acordo com as suas necessidades. Outras temáticas debatidas foram propostas pelas acadêmicas de enfermagem do Projeto, com base em uma análise prévia e diagnóstico da situação. Mediante isso, os temas discutidos foram: saúde mental do profissional, escuta sensível durante as visitas domiciliares, calendário vacinal, preenchimento e importância da caderneta da criança, entre outros. Avaliaram-se essas ações como de grande importância e essenciais, visto que houve grande assiduidade e participação dos profissionais durante as atividades.

Apesar de reconhecer certa organização e funcionamento adequado dos Programas de Saúde, bem como o profissionalismo e dedicação dos profissionais que atuam na Unidade, observou-se pontos de melhoria como a necessidade de 
implantação do Programa de Atenção Integrada às Doenças Prevalentes na Infância (AIDPI), realização de testes de triagem neonatal em todas as Unidades, deficiência no quantitativo de profissionais impossibilitando a realização de algumas atividades.

Entretanto, avalia-se a participação enquanto alunos de curso de graduação como essencial para a formação e muito importante para a segurança enquanto futuro profissional atuante no sistema de saúde, mais especificamente na Atenção Básica. A atuação no Multicampi possibilita a vivência diária e qualificação da prática profissional.

Corroborando com isso, Pessoa et al. (2018) aponta a necessidade de discussão da articulação entre as universidades e os serviços de saúde para a mudança na formação em saúde. Esta associação permite a compreensão das necessidades de saúde da população, além de direcionar a formação para o SUS, que será novamente beneficiado com a inserção desses futuros profissionais na rede de serviços.

Para Moreira et al. (2018), a base da construção da carreira profissional do egresso de enfermagem está atrelada à formação acadêmica. Para isso, a adequada interação teórico/prática faz-se de grande importância, pois, além do crescimento pessoal adquirido, o acadêmico utiliza os conteúdos e práticas transmitidos pela instituição de ensino como subsídio para sua inserção e adaptação no mercado de trabalho.

A atuação prática, pelo período e moldes do Projeto, estimula a reflexão crítica construtiva do discente e planejamento de atividades que possua a complexidade que o sistema e a comunidade exigem. Além de que, compreendendo o cuidado como complexo e com necessidade de um trabalho interdisciplinar, incentiva, ainda mais a busca de resolução dos problemas da comunidade e famílias de modo a aproximar o graduando da equipe de saúde e demais profissões da saúde e afins.

Para Mendes et al. (2020), a integração entre ensino e serviço proporciona melhor capacitação do docente, do estudante e do profissional do serviço de saúde. Por conseguinte, garante ações e serviços de qualidade à população, por meio da reorientação da atenção básica e do modelo de atenção à saúde vigente no sistema nacional. Esta integração também contribui para fortalecer a formação capacitada do estudante para atuar em diferentes cenários de atenção à saúde, incrementar o processo de capacitação do profissional em serviço, assim como promover o trabalho multiprofissional em todos os níveis do sistema.

Ao final das atividades do Multicampi, cada acadêmico deve apresentar os relatórios de atividades, diários de campo e um seminário demonstrando as demandas da criança-guia elegida e sob sua responsabilidade, bem como o planejamento, intervenções realizadas e resultados atingidos.

Os discentes são avaliados pelos seus respectivos preceptores quanto sua participação no Projeto no que diz respeito às atividades realizadas, produtos elaborados, planejamento e execução de ações, capacitações e ações educativas realizadas, além de aspectos como interesse, assiduidade, pontualidade, criatividade, capacidade de liderar e qualidade do serviço aplicado. Essa avaliação também proporciona uma auto-avaliação crítica reflexiva capaz de contribuir com a formação acadêmica e profissional.

Portanto, diante da vivência no Projeto Multicampi Saúde, entende-se a integração ensino-serviço e comunidade como uma importante estratégia para alcançar as mudanças no processo formativo dos acadêmicos e futuro profissionais de saúde, possibilitando modificações nas práticas profissionais ocupacionais e no modelo de assistência, contribuindo para melhorias na qualidade e na oferta de ações oferecidas à comunidade. 


\section{Considerações Finais}

O projeto teve grande importância para as acadêmicas, para o serviço e para a comunidade, pois foi possível utilizar de variados conhecimentos (teórico/prático/científico) para a execução de cada atividade desenvolvida. A oportunidade de encarar desafios com o serviço e a comunidade, possibilitou a colaboração das acadêmicas no serviço a desenvolver novas tecnologias para facilitar o atendimento e conscientização da comunidade ao que se refere a sua saúde de maneira integral e familiar.

O trabalho multiprofissional desenvolvido no Projeto possibilitou um atendimento multidimensional dos usuários e suas famílias, alcançando o objetivo de prestar um atendimento à saúde mais eficaz e resolutivo.

Através das visitas domiciliares, pudemos atender as famílias e adentrar a sua realidade, sendo essenciais para o tratamento continuado integral, tanto da criança-guia, quanto da família como um todo. Utilizando de atividades lúdicas junto às crianças e estímulos para o restante dos familiares a executarem o planejamento proposto pelas acadêmicas junto à equipe multiprofissional.

Desta forma, diante de todas as atividades realizadas, análise e publicação de seus resultados e desfecho foi possível compreender a grande valia do Projeto para a comunidade, profissionais, acadêmicos e ciência.

O grande desafio do projeto Multicampi Saúde é fazer com que os discentes compreendam a importância do trabalho em equipe dentro da unidade de saúde em que atua e que possam estabelecer integração com as demais Unidades da rede para garantir o princípio da integralidade do SUS. Nesse sentido, ocorre a necessidade de criar mais espaços durante a graduação para que a integração ensino-serviço não seja apenas pensada e discutida, mas também executada e vivenciada pelos discentes.

Nesse sentido, considera-se importante a realização de futuras pesquisas que possam revelar a experiência de discentes e docentes envolvidos em projetos de extensão universitárias, sejam elas do tipo relato de experiência ou pesquisas de campo. Além disso, permitir um estudo mais aprofundado sobre a visão de enfermeiros no processo de formação acadêmica diante de experiências com usuários do SUS e cuidado à comunidade para contribuição na construção do conhecimento pessoal e profissional.

\section{Referências}

Brito, G. V., Albuquerque, I. M. N. A., Ribeiro, M. A., Ponte, E. S., Moreira, R. M., \& Linhares, M. C. (2018). Consulta de puericultura na estratégia saúde da família: Percepção de enfermeiros. Revista de APS, 21(1). https://doi.org/10.34019/1809-8363.2018.v21.16040

Brito, M. C., Teófilo, F. S., Dias, M. A., Vasconcelos, M. O., Albuquerque, I. N., \& Silva, L.C. (2018). Formação do enfermeiro para a atenção básica: Um olhar sobre o conhecimento produzido. Sanare - Revista de Políticas Públicas, 16(2). 10.36925/sanare.v16i2.1183.

Daltro, M., \& Faria, A. (2020). Relato de experiência: Uma narrativa científica na pós-modernidade. Estudos e Pesquisas em Psicologia, 19 (1), $223-237$. http://pepsic.bvsalud.org/pdf/epp/v19n1/v19n1a13.pdf.

Ferreira, L., Barbosa, J., Esposti, C., \& Cruz, M. (2019). Educação Permanente em Saúde na atenção primária: Uma revisão inte grativa da literatura. Saúde em Debate, 43(120), 223-239. 10.1590/0103-1104201912017.

Ferreira, S., Périco, L., \& Dias, V. (2018). The complexity of the work of nurses in Primary Health Care. Revista Brasileira de Enfermagem, 71(1), 704-709. https://doi.org/10.1590/0034-7167-2017-0471.

Justus Neto, A., Bernardi, L., \& Novello, D. (2017). Trabalho interdisciplinar entre profissionais de equipes de Estratégia Saúde da Família das $4^{\mathrm{a}}$ e $5^{\mathrm{a}}$ Regionais de Saúde do Paraná, Brasil: Uma investigação qualitativa. Revista Spacios, $38(42)$, 26. https://www.revistaespacios.com/a17v38n42/a17v38n42p26.pdf.

Lei $\mathrm{n}^{\circ}$ 7.498, de 25 de Junho de 1986. Dispõe sobre a regulamentação do exercício da Enfermagem e dá outras providências. http://www.planalto.gov.br/ccivil_03/leis/17498.htm.

Lopes, O., Henriques, S., Soares, M., Celestino, L., \& Leal, L. (2020). Competências dos enfermeiros na estratégia Saúde da Família. Escola Anna Nery, 24(2). https://doi.org/10.1590/2177-9465-ean-2019-0145.

Menezes, L., Ciuffo, L., Gonçalves, A., Moraes, J., Souza, T., \& Rodrigues, E. (2019). A criança e sua família na atenção primária em saúde. Revista de Enfermagem UFPE On Line, 13. 10.5205/1981-8963.2019.241426. 
Ministério da Saúde. Gabinete do Ministro. Portaria no 1.130, de 05 de Ago sto de 2015. Institui a Política Nacional de Atenção Integral à Saúde da Criança (PNAISC) no âmbito do Sistema Único de Saúde (SUS). Diário Oficial da União, Brasília-DF. http://bvsms.saude.gov.br/bvs/saudelegis/gm/2015/prt1130_05_08_2015.html.

Ministério da Saúde. Gabinete do Ministro. Portaria n 2.436, de 21 de setembro de 2017. Aprova a Política Nacional de Atenção Básica, estabelecendo a revisão de diretrizes para a organização da Atenção Básica, no âmbito do Sistema Único de Saúde (SUS). Diário Oficial da União, Brasília-DF. https://bvsms.saude.gov.br/bvs/saudelegis/gm/2017/prt2436_22_09_2017.html.

Miyake, R. (2020, 04 de dezembro). UFPA tem novo projeto voltado para o atendimento à comunidade: o Multicampi Saúde. Jornal Beira do Rio. https://www.portal.ufpa.br/index.php/ultimas-noticias2/10408-ufpa-tem-novo-projeto-voltado-para-o-atendimento-a-comunidade-o-multicampi-saude.

Moreira, L. R., Siqueira, A. T., Santos, P. T., \& Ladislau, V. N. (2018). Percepção do enfermeiro acerca da formação acadêmica para o exercício profissional. Enfermagem Revista, 21 (1), 34-50. http://C:/Users/lical/Downloads/17896-Texto\%20do\%20artigo-63889-1-10-20180703\%20(2).pdf.

Pereira, A. S., Shitsuka, D. M., Parreira, F. J. \& Shitsuka, R. (2018). Metodologia da pesquisa científica. Santa Maria, RS: UFSM, NTE, 2018.

Rocha, B., Guerra, D., Maia, L., Lima, L., Silva, D., \& Silva, L. (2020). Trabalho da equipe multiprofissional na atenção primária à saúde. Propostas, Recursos e Resultados nas Ciências da Saúde 5, 161-166.10.22533/at.ed.31220240619.

Sousa, P., Souza, R., Costa, M., Azevedo, M., Torres, R., \& Nascimento, G. (2020). Protagonismo do enfermeiro na atenção básica de saúde/nursing protagonism in basic health care. Brazilian Journal of Development, 6(10), 76244-76257. 10.34117/bjdv6n10-157. 